\title{
Molecular mechanisms of humic substances stabilization
}

\section{Chukov S.N.}

Saint Petersburg State University, Russia, S.Chukov@spbu.ru

Keywords: humification, humic acids, 13C-NMR, polyconjugated aromatic structures

doi: 10.36291/HIT.2019.chukov.025

Humic substances (HS) are one of the largest reservoirs of carbon accumulation in the biosphere. This becomes possible due to their high biochemical stability, which is acquired in the process of humification. The study of the molecular mechanisms of stabilization of HS in soils and other natural sites is one the fundamental problems of natural science.

An important factor in the physical and biochemical stability of HS is the presence in their composition of the cyclic type fragments and especially polyconjugated (condensed) aromatic structures. From the point of view of biothermodynamic and biochemical stability, cyclic structures (aromatic and heterocyclic) possess the greatest stability. Their content relatively increases with the depth of humification.

The mechanism of the formation and accumulation of polyconjugate aromatic structures in the soils HS molecules is still unclear. In the work of Lishtvan et al. (2012) revealed the formation of aromatic polyconjugation systems by destroying molecular fragments of an aliphatic structure and polymerization of monoaromatic fragments. At the stage of the genesis of brown coal, when there is a sharp decrease in the content of monoaromatic fragments and an increase in the content of polyconjugation systems, there is also an increase in the size of "planar" graphite-like structures (Lishtvan et al. 2012).

In our studies, it was shown by solid-state 13C-NMR spectroscopy in humic acids (HAs) of soils with a large degree of humification (chernozems and gray forest soils), there are poly-conjugated aromatic structures of the "coal" type. But we did not find any polyconjugated aromatic structures in the soils HA of the taiga zone (the aromatic part is represented mainly by "scattered" nuclei). Apparently, in the HA of chernozems, we are dealing with purely soil biochemical processes of the formation of "humus coal". Many researchers attribute this stable organic substance to the fraction of humins associated with clay minerals (Krasilnikov, 2016; Wang e.a., 2014). But the discovery of such structures in HA preparations isolated from humus horizons by pyrolysis mass spectrometry (Schulten and Schnitzer, 1995) and NMR (Chukov, 1998, 2018; Nicole DiDonato, EA, 2017, etc.) indicates that the processes of formation of such stable compounds occur during humification during the formation of the "nuclear" part of HA macromolecules.

To decipher the molecular mechanisms of the formation of polyconjugate aromatic structures, it is necessary to study the HS in both natural and experimental conditions. The study of the HS of soils contrasting in the biohydrothermal conditions of humus formation, as well as the study of the "further" humification and decarbonization of HS in model laboratory experiments. 